\title{
Inflammatory Breast Cancer: Dynamic Contrast-enhanced MR in Patients Receiving Bevacizumab. Initial Experience
}

\author{
Arpi Thukral,MD ${ }^{1}$ \\ David M. Thomasson, $\mathrm{PhD}^{1}$ \\ Catherine K. Chow,MD ${ }^{1}$ \\ Reyes Eulate,MD ${ }^{1}$ \\ Suparna B. Wedam,MD ${ }^{1}$ \\ Sandeep N. Gupta, $\mathrm{PhD}^{1}$ \\ Betty J. Wise, BS, RT(R)MR ${ }^{1}$ \\ Seth M. Steinberg, $\mathrm{PhD}^{1}$ \\ David J. Liewehr, $\mathrm{PhD}^{1}$ \\ Peter L. Choyke,MD ${ }^{1}$ \\ Sandra M. Swain, MD ${ }^{2}$
}

${ }^{1}$ From the Medical Oncology Branch (A.T., S.B.W., S. M. Swain), Molecular Imaging Program (R.E., P.L.C.), and Biostatistics and Data Management Section (S. M. Steinberg, D.J.L.), Center for Cancer Research, National Cancer Institute; and Diagnostic Radiology Department, Warren G. Magnuson Clinical Center (D.M.T., C.K.C., B.J.W.), National Institutes of Health, 8901 Wisconsin Ave, Bldg 8, Room 5101, Bethesda, MD 20889-5015; and GE Healthcare Technologies, Hanover, Md (S.N.G.).

2 Current address: Washington Cancer Institute, Washington Hospital Center, Washington, D.C.

Address correspondence to S. M. Swain (Sandra.M.Swain@Medstar.net). 


\section{PURPOSE}

To retrospectively compare three dynamic contrast material-enhanced magnetic resonance (MR) imaging (dynamic MR imaging) analytic methods to determine the parameter or combination of parameters most strongly associated with changes in tumor microvasculature during treatment with bevacizumab alone and bevacizumab plus chemotherapy in patients with inflammatory or locally advanced breast cancer.

\section{MATERIALS AND METHODS}

This study was conducted in accordance with the institutional review board of the National Cancer Institute and was compliant with the Privacy Act of 1974. Informed consent was obtained from all patients. Patients with inflammatory or locally advanced breast cancer were treated with one cycle of bevacizumab alone (cycle 1) followed by six cycles of combination bevacizumab and chemotherapy (cycles 2-7). Serial dynamic MR images were obtained, and the kinetic parameters measured by using three dynamic analytic MR methods (heuristic, Brix, and general kinetic models) and two region-ofinterest strategies were compared by using two-sided statistical tests. A $P$ value of .01 was required for significance.

\section{RESULTS}

In 19 patients, with use of a whole-tumor region of interest, the authors observed a significant decrease in the median values of three parameters measured from baseline to cycle 1: forward transfer rate constant $\left(K^{\text {trans }}\right)$ ( $\_34 \%$ relative change, $\left.P=.003\right)$, backflow compartmental rate constant extravascular and extracellular to plasma $\left(K_{\text {ep }}\right)$ $(-15 \%$ relative change, $P<.001)$, and integrated area under the gadolinium concentration curve (IAUGC) at 180 seconds ( $-23 \%$ relative change, $P=.009)$. A trend toward differences in the heuristic slope of the washout curve between responders and nonresponders to therapy was observed after cycle 1 (bevacizumab alone, $P=.02$ ). The median relative change in slope of the wash-in curve from baseline to cycle 4 was significantly different between responders and nonresponders $(P=.009)$.

\section{CONCLUSION}

The dynamic contrast-enhanced MR parameters $K^{\text {trans }}, K_{\text {ep }}$, and IAUGC at 180 seconds appear to have the strongest association with early physiologic response to bevacizumab. 


\section{INTRODUCTION}

Dynamic contrast material-enhanced magnetic resonance (MR) imaging (dynamic MR imaging) can depict changes in the physiologic characteristics of tumors after a therapeutic intervention. Dynamic MR imaging has been used to monitor response to chemotherapy and for drug development; therefore, it may be clinically useful for determining prognoses (1-3). Bevacizumab (Avastin; Genentech, South San Francisco, Calif) is a recombinant humanized monoclonal antibody that binds specifically to and inhibits vascular endothelial growth factor $(4,5)$. Because vascular endothelial growth factor is involved in the proliferation and differentiation of endothelial cells, it is an attractive intervention target that could be monitored with dynamic MR imaging.

Conventional anatomic imaging depicts the physical size of tumors and is therefore considered a delayed indicator that might not enable reliable prediction of outcome. Use of dynamic MR imaging to characterize the tumor microvasculature is attractive because the technique is easy to implement, involves no radiation exposure (allowing repeated use), and generates semiquantitative information about vascular permeability and blood flow within tumors (6). Because dynamic MR imaging signal kinetics correspond to a tumor's vascular parameters, quantification of these signals may be used to evaluate response to angiogenic inhibition $(1,3)$. The pathophysiologic basis for these contrast material kinetics has been attributed to the hyperpermeability of angiogenic vessels (7).

Compartment models such as the Brix model and the general kinetic model (GKM) $(8,9)$, which are used to calculate the leakage of contrast material from the vascular space to the extravascular-extracellular space (leak rates) and the reflux of contrast material back to the vascular space (reflux rate), have been established. Previous dynamic MR imaging studies to examine the effects of anti-vascular endothelial growth factor treatment in athymic rats with human breast carcinoma xenografts have revealed decreases in fractional leak rates and reflux rates, compared with these rates in control animals, as early as 24 hours after treatment (10). Similar results were obtained with other preclinical models of ovarian cancer assessed with dynamic MR imaging (11).

In a previous clinical trial (12), we observed substantial decreases in pharmacokinetic parameters during treatment in patients with previously untreated inflammatory or locally advanced breast cancer who underwent one cycle of bevacizumab as a monotherapy (cycle 1) followed by six cycles of combination bevacizumab, doxorubicin, and docetaxel therapy (cycles 2-7). Thus, the purpose of our study was to retrospectively compare three dynamic MR imaging analytic methods to determine the parameter or combination of parameters most strongly associated with changes in tumor microvasculature during treatment with bevacizumab alone and bevacizumab plus chemotherapy in patients with inflammatory or locally advanced breast cancer.

\section{MATERIALS AND METHODS}

\section{Clinical Trial}

The data from a trial to evaluate the effects of bevacizumab, doxorubicin, and docetaxel in patients with previously untreated locally advanced or inflammatory breast cancer 
were analyzed (12). The trial was approved by the institutional review board of the National Cancer Institute and compliant with the Privacy Act of 1974. Eligible patients had stage III or IV inflammatory breast cancer or locally advanced breast cancer, which included stages IIB, IIIA, IIIB, and IIIC tumors (13). Signed informed consent was obtained from all patients before the initiation of treatment. The patients underwent a total of seven cycles of treatment: one cycle of therapy with bevacizumab (15 mg per kilogram of body weight) followed by six cycles of combination bevacizumab (15 $\mathrm{mg} / \mathrm{kg}$ ), doxorubicin $(50 \mathrm{mg} / \mathrm{m} 2)$, and docetaxel $(75 \mathrm{mg} / \mathrm{m} 2)$ therapy, with a 3-week interval between cycles. All patients underwent dynamic MR imaging at baseline (ie, before therapy) and after therapy cycles 1, 4, and 7, 3 weeks after a cycle and no more than 2 days before the next cycle of chemotherapy or, in the case of the last cycle of chemotherapy, before definitive surgery. The last MR examination was performed 3 weeks after the last cycle of chemotherapy and 4-6 weeks before definitive surgery to confirm the response to therapy.

Measurable disease was quantified by using MR imaging according to the response criteria in solid tumors (RECIST) guidelines (14). The index lesion was the primary breast mass (if discrete), the enlarged axillary node, or both. One patient's axillary adenopathy was followed up with computed tomography because of the limited field of view at MR imaging. Disease response was assessed by the same reader (C.K.C.) by using the sequence that revealed the abnormality most clearly. For assessment of the lymph nodes, the non-fat-suppressed dynamic sequence (see Dynamic Contrastenhanced MR Imaging Analysis) was used, whereas for assessment of the primary breast mass, measurements were obtained by using the contrast-enhanced fast spoiled gradientecho sequence. According to RECIST criteria, the longest axial dimension was recorded regardless of the orientation. Residual disease was assessed with reference to the original prechemotherapy images. (Also see Region-of-interest [ROI] selection in Dynamic Contrast-enhanced MR Imaging Analysis section.) Patients were divided into responders, who had a partial response to therapy, and nonresponders, who had stable or progressive disease after therapy (12).

\section{MR Imaging Techniques}

Imaging was performed with patients in the prone position by using a 1.5-T MR system (GE Healthcare, Waukesha, Wis) with a dedicated receive-only four-channel dual breast coil. Baseline transverse gradient-echo images were obtained with a $25-35-\mathrm{cm}$ field of view set to encompass both breasts and the axilla. First, diagnostic T2-weighted images were obtained by using 5225/100 (repetition time msec/echo time msec), a section thickness of $5 \mathrm{~mm}$, and a matrix of $128 \times 256$ pixels. Next, dynamic contrast-enhanced MR images were obtained with a three-dimensional spoiled gradient-echo sequence by using 8/4.2, a $25^{\circ}$ flip angle, 4-5-mm-thick sections through the entire breast, an acquisition time of 30 seconds per data set, and a matrix of $128 \times 256$ pixels. After three baseline nonenhanced image acquisitions, an automatic injector (Medrad Spectris, Indianola, $\mathrm{Pa}$ ) was used to intravenously infuse gadopentetate dimeglumine (Magnevist; Berlex Laboratories, Wayne, $\mathrm{NJ}$ ) at $0.3 \mathrm{~mL} / \mathrm{sec}$, for a total of $0.1 \mathrm{mmol}$ per kilogram of body weight (typically 15-20 mL), followed by a 50-mL normal saline flush. The 0.3 $\mathrm{mL} / \mathrm{sec}$ infusion rate was chosen to satisfy the Brix model. Continuous 30 second imaging data sets were obtained before, during, and after administration of the contrast medium for a total of 8 minutes to result in 20 repeated data sets. 


\section{Dynamic Contrast-enhanced MR Imaging Analysis}

We compared three dynamic MR imaging analytic methods - the heuristic, Brix (8), and GKM (9) techniques and two approaches to defining the ROI (10). The analysis and ROI selection were performed by three authors (A.T., R.E., and B.J.W.) with 1-6 years experience in breast MR imaging. These three methods were chosen because they represent three approaches to modeling: With the heuristic approach, one makes no assumption about tissue compartmentalization, and the two methods based on tissue compartmentalization differ in terms of the assumptions made about the influence of measured (GKM technique) versus modeled (Brix technique) arterial input function on the kinetic parameter values. The results of these three methods were obtained by transferring the acquired images to a personal computer and processing them with two analysis programs that were developed in house and based on the interactive display language (IDL, Boulder, Colo): the Dynamic program for the Brix and heuristics methods and the Cinetool program (GE Healthcare) with a KinMode analysis module for the GKM method. The GKM computer model was provided by one of the authors (S.N.G.), who programmed the module into the Cinetool research software.

The time-signal intensity data from each pixel on the image generated their own timesignal intensity curves, which were then evaluated according to the dynamic MR imaging method used with the heuristic model or one of the pharmacokinetic models (Brix or GKM) (15). The parameters used for the heuristic model were direct measurements of the slopes of wash-in and washout curves and the integrated area under the gadolinium concentration curve (IAUGC) for the first 90 or 180 seconds after contrast material injection. The parameters derived for the Brix model were the amplitude of enhancement and the reverse transfer constant (8). The parameters derived for the GKM method were the forward transfer rate constant $\left(\mathrm{K}^{\text {trans }}\right)$, the backflow compartmental rate constant extravascular and extracellular to plasma $\left(\mathrm{K}_{\mathrm{ep}}\right)$, and the extravascular-extracellular volume fraction, with the assumption of a mean parenchyma T1 value of $850 \mathrm{msec}$. These rate transfer constants were originally described by Kety (9) and were modified to incorporate two-compartment models $(9,16,17)$. Arterial input functions were obtained by drawing an ROI around the aorta from a single central section.

ROI selection. Because there is no standardized method of plotting or summarizing the data within an ROI, we used two techniques to determine which strategy yielded the most clinically meaningful results. First, we chose an ROI from a single section of the most enhanced area of the tumor - that is, we performed "hot spot" measurements (18). These ROIs were hand drawn on color maps created with the Brix model and then exported to the two other models. Second, we used a whole-tumor pixel-by-pixel averaging technique: The software automatically drew the ROI by using a regiongrowing algorithm bounded by an assigned threshold value, encompassing approximately $90 \%$ of the tumor area. Whole-tumor regions included only numerical data from pixels with a goodness-of-fit index ( $R 2$ value) of at least 0.85 , which effectively eliminated nonenhancing or poorly enhancing regions. We chose this technique on the basis of findings in a prior study that revealed no apparent advantage in subsampling tumor regions (19).

Statistical analyses. In the current analysis, we used data from a single-arm, singlestage study designed for enrollment of 20 examinable patients to achieve $95 \%$ power for 
the detection of a change in any of four parameters measured from baseline to the end of cycle 1 , equal to 1 standard deviation of the change. The two-tailed Wilcoxon signed rank test (or t test when appropriate) with an a level of .05 was used to perform these analyses.

The primary outcomes of these analyses were changes in each dynamic MR imaging parameter. Initial explorations indicated that actual differences between the baseline measurement and the cycle 1, 4, or 7 measurement were more dependent on the baseline value (B) than were the corresponding relative differences - for example, the relative difference of $(\mathrm{C} 1-\mathrm{B}) / \mathrm{B}$ for each parameter, where $\mathrm{C} 1$ is the cycle 1 value. Thus, these relative differences, converted into percentages, were used as the primary data for the analyses since the evaluation of absolute differences might have revealed greater bias as a function of the magnitude of the baseline values themselves.

To test whether the percentage relative changes were associated with a statistically significant difference from zero, the Wilcoxon signed rank test was used. Comparisons between responders and nonresponders were performed purely as secondary exploratory evaluations with low power by using the exact Wilcoxon rank sum test.

Because of the large number of parameters evaluated, the various degrees of independence from one parameter to another, and the exploratory nature of the study, to interpret results in the context of the multiple comparisons performed, only $\mathrm{P}$ values of less than .01 were considered to indicate statistical significance and $\mathrm{P}$ values of .01-.05 were associated with trends. All reported $\mathrm{P}$ values were de-rived by using two-sided tests and are presented without adjustments for multiple comparisons. Analyses were performed by using 2001 SAS, version 8.2, software (SAS Institute, Cary, NC).

\section{RESULTS}

\section{Patient Characteristics}

Twenty-one women aged 35-73 years (mean age, 51 years; median age, 50 years) were enrolled in the initial trial from October 1, 2001, to May 31, 2004. All enrolled patients underwent at least the first cycle of bevacizumab (cycle 1). One patient had noninflammatory locally advanced breast cancer. For the current analyses, the data of 19 of the 21 patients were used. Dynamic contrast-enhanced MR imaging was performed in 20 patients at baseline, in 18 patients after cycle 1, in 19 patients after cycle 4 , and in 15 patients after cycle 7 . Reasons for not completing all four MR examinations included the following: The patient size was too large, the tumor was too large and/or the procedure was too painful to tolerate, intravenous access could not be gained to perform the dynamic portion of the examination, or the patient was removed from the study. Longitudinal tumor dimensions ranged from 1.9 to $8.4 \mathrm{~cm}$. To compare the change in dynamic MR imaging parameters according to clinical response (based on RECIST criteria), we assigned the 19 patients to a responder or nonresponder group. Thirteen patients were considered clinical responders (including one whose partial response was unconfirmed because surgery was performed before reassessment), and six were considered nonresponders (ie, patients with stable or progressive disease after 
therapy). One patient with a clinically partial response had a complete pathologic response. There were no clinically complete responders.

\section{Dynamic Contrast-enhanced MR Imaging Results}

Serial GKM K ${ }^{\text {trans }}$ parametric maps and gadolinium concentration-time curves (Fig 1) showed the distribution of high-vascular-permeability surface areas. A high degree of vascular permeability was observed on the initial maps (constructed at baseline and after cycle 1), but less vascular permeability was observed on the subsequent maps (constructed after cycles 4 and 7). The kinetics of gadolinium enhancement in the tumor, as shown in concentration-time curves, were presented for each map. The higher initial gadolinium concentration slope at baseline and after cycle 1 reflected increased compartmental wash-in of the contrast agent, and the time points after the wash-in peak reflected the compartmental outflow or washout.

Longitudinal data (ie, baseline, cycle 1, cycle 4, and cycle 7 measurements) for eight parameters of the three analytic models - Brix amplitude of enhancement, Brix reverse transfer constant, slope wash-in, slope washout, IAUGC at 90 seconds, IAUGC at 180 seconds, GKM K ${ }^{\text {trans }}$, and GKM K $\mathrm{ep}_{\mathrm{ep}}$ - were collected for all 19 patients who underwent MR imaging. Note that slope washout is the only parameter that can assume either a positive or a negative value, and it can change from positive to negative-and vice versa-after therapy. Also, because very small slope washout values were measured at base-line, modest absolute value changes could have led to substantial percentage changes in value relative to the baseline measurement.

The initial results obtained by using ROIs based on hand-drawn areas around the most enhanced area of the tumor (ie, hot spot) indicated trends toward differences in the heuristic parameters slope wash-in $(\mathrm{P}=.04)$, slope washout $(\mathrm{P}=.02)$, and IAUGC at 180 seconds $(\mathrm{P}=.049)$ between the responders and the nonresponders. Results also indicated trends toward differences in the GKM parameters $\mathrm{K}^{\text {trans }}$ and $\mathrm{K}_{\mathrm{ep}}(\mathrm{P}>.01$ for both). In addition, when the relative differences in measurements obtained from baseline to cycle 1 were examined according to response, only the values for the heuristic IAUGC at 90 seconds $(\mathrm{P}=.05)$ and the slope washout $(\mathrm{P}=.05)$ differed. For measurements obtained from baseline to cycle 4, there was a trend toward differences in the heuristic IAUGC at 180 seconds only $(\mathrm{P}=.03)$. There were no significant differences.

At whole-tumor analysis, the results were considerably different (Table 1). Three parameters had a significant decrease as the relative change between baseline and cycle 1: GKM K ${ }^{\text {trans }}$ (median relative change, $-34 \% ; \mathrm{P}=.003$ ), $\mathrm{K}_{\mathrm{ep}}$ (median relative change, $-15 \% ; \mathrm{P}<.001$ ), and IAUGC at 180 seconds (median relative change, $-23 \% ; \mathrm{P}=.009$ ). Between baseline and cycle 4 and between baseline and cycle 7, almost all parameters of the three models decreased (Fig 2, Table 1). For all parameters measured, the decrease in value was greater earlier in therapy (between cycles 1 and 4) than later in therapy (between cycles 4 and 7) (Table 2). We observed one trend toward a difference between the responders and the nonresponders when we compared the relative changes in all eight parameters from baseline to cycle 1 and from baseline to cycle 4: that for the median relative change in slope washout from baseline to cycle 1 ( $-104 \%$ for responders, $65 \%$ for nonresponders $[\mathrm{P}=.02]$ ) (Table 3 ). Although the median relative change in slope wash-in from baseline to cycle 4 was significantly different between the 
responders $(-60 \%)$ and the nonresponders $(-31 \%)(\mathrm{P}=.009)$, there was no significant difference in this parameter between the two groups from baseline to cycle 1 (Fig 3).

\section{DISCUSSION}

In this analysis, we found that after a single cycle of bevacizumab, all parameters of the three analytic methods decreased, suggesting that these parameters reflect vascular changes. However, only the GKM parameters $\mathrm{K}^{\text {trans }}$ and $\mathrm{K}_{\mathrm{ep}}$ and the heuristic model parameter IAUGC at 180 seconds were significantly different from the baseline values. A second, exploratory goal of the study was to determine whether dynamic contrastenhanced MR imaging might reveal an earlier change that could be associated with clinical outcome. After combination bevacizumab therapy and chemotherapy, the parameters of all methods decreased substantially. The possibility that changes in these parameters also result from prolonged and/or additional bevacizumab therapy cannot be ruled out; however, it is more likely that the combination of the angiogenic inhibitor and the chemotherapy had additive effects. The parameter values measured between cycles 1 and 4 showed greater differences than did those measured between cycles 4 and 7, suggesting that the greatest effect on tumor microvascularity occurred early in the course of therapy.

Although none of the assessed methods enabled successful prediction of the clinical response after cycle 1, we observed a significant difference in the heuristic slope washin between the responders and the nonresponders at cycle $4(\mathrm{P}=.009)$. This finding suggests that choosing the appropriate time to obtain dynamic MR imaging data may improve the results. However, we speculate that this parameter was the only one that enabled differentiation between the two groups because of the change in tumor volume throughout the course of treatment and because semiquantitative calculations are less sensitive to noise from heterogeneous tissues.

Our results indicate that methods in which arterial input functions are incorporated into the pharmacokinetic model or in which there is no attempt at physiologic correlation are the most reproducibly sensitive to angiogenic response to therapy. In that regard, our results from the heuristic model are consistent with previous results, which show that although the coefficient of variation for simple signal slopes (gradients) is higher than that for similar quantitative methods, IAUGC values may have a lower coefficient of variation than do other quantitative parameters (20). At comparisons of other heuristic parameters, such as maximal signal intensity change per time interval ratio and time to enhancement (time at which the signal intensity reaches $90 \%$ of its maximum), it has been shown that these parameters have a higher coefficient of variation (21).

In our study, the significance of the IAUGC likely resulted from the fact that simple signal integration is more accommodating of "noisy" data and less sensitive to data heterogeneity. Although the IAUGC is sensitive to change, it is limited because it cannot enable differentiation of the shape of various enhancement patterns. For example, a simple continuous increase in signal intensity can yield the same IAUGC value as a sharp increase and rapid decrease that is characteristic of angiogenesis enhancement patterns. Confirmation and validation of these findings in larger studies are needed. 
Another important part of our analysis was the selection of the ROI on the MR image. We used the hot-spot and whole-tumor ROI selection methods. In a similar breast tumor study (18), a hot-spot method yielded results that were more useful than whole-tumor ROI method results for the differentiation of benign versus malignant disease (18). Although the results of another hot-spot technique indicated a larger difference between responders and nonresponders in a breast cancer neoadjuvant chemotherapy study (22), these results possibly were skewed because the whole-tumor data included the signal intensity of nonenhancing necrotic areas. In our evaluation, use of the hot-spot ROI method did not enable optimal detection of the small subtle changes that result from antiangiogenic therapy; however, when the whole tumor was evaluated, sensitivity improved. Moreover, determinations of the whole-tumor ROI were less subjective (23). Unlike the two previously described hot-spot methods, in which the analysis could have been biased owing to necrotic areas of the tumor, the ROI drawn by our automated ROI-drawing tool in the software supplied with the GKM algorithm included only numerical data from pixels with a goodness-of-fit index (R2) of at least 0.85 , which effectively eliminated the nonenhancing or poorly enhancing regions.

Our study had several limitations: First, because of the rarity of inflammatory breast cancer, the study included data from a small number of patients. Second, because invasive tumor cells in inflammatory breast cancer are distributed more heterogeneously throughout the affected breast tissue, discrete tumor measurement is more challenging. Despite this problem, we were able to identify important changes in dynamic MR imaging parameters after bevacizumab treatment. Our third limitation was due to the study design: Because the patients received only one cycle of bevacizumab monotherapy before undergoing combination chemotherapy, the information regarding the effects of bevacizumab alone was limited. To be conservative, we required a stricter interpretation of the level of significance associated with a given $\mathrm{P}$ value because of the large number of tested parameters. Other methods in subsequent analyses may involve parameters that are predictive if they are examined as single parameters. Finally, we were restricted to using a low contrast material infusion rate $(0.3 \mathrm{~mL} / \mathrm{sec})$ to accommodate the requirements of the Brix model. It is unknown whether faster bolus infusion would have produced different results.

In conclusion, our study results show that dynamic contrast-enhanced MR imaging can be used to reliably detect and characterize the effects of the angiogenesis inhibitor bevacizumab. We found that the MR-derived IAUGC and GKM parameters $\mathrm{K}^{\text {trans }}$ and $\mathrm{K}_{\mathrm{ep}}$ were the most strongly associated with changes in inflammatory breast cancer response after treatment with bevacizumab alone and combination bevaci-zumab therapy and chemotherapy. This may be because the GKM parameters are based on independent measurements of arterial input function and T1 correction-rather than MR signal intensity alone-for determination of gadolinium concentrations. Thus, the physiologic characteristics of individual patients are taken into account in these values. With continued improvements in dynamic contrast-enhanced MR imaging, such as faster acquisitions, improved postprocessing algorithms, and improved ROI analysis, we expect this method to contribute valuable prognostic information for angiogenic therapy. 


\section{ADVANCES IN KNOWLEDGE}

MR-derived general kinetic model pharmacokinetic parameters and the integrated area under the gadolinium concentration curve are statistically significant methods of detecting changes in the response of inflammatory breast cancer to bevacizumab alone and bevacizumab combined with chemotherapy.

The whole-tumor region-of-interest selection method with suitable thresholding yields better statistical correlations for parameter changes from baseline to cycle $1(\mathrm{P}=.003$ for forward transfer rate constant $\left[\mathrm{K}^{\text {trans }}\right], \mathrm{P}<.001$ for backflow compartmental rate constant extravascular and extra-cellular to plasma $\left[\mathrm{K}_{\mathrm{ep}}\right], \mathrm{P}=.009$ for integrated area under the gadolinium concentration curve [IAUGC] at 180 seconds) than does the hotspot method ( $\mathrm{P}>.01$ for $\mathrm{K}$ trans and $\mathrm{K}_{\mathrm{ep}}, \mathrm{P}=.049$ for IAUGC at 180 seconds).

\section{IMPLICATIONS FOR PATIENT CARE}

Dynamic contrast-enhanced MR imaging may be a useful tool for assessment of vascularity changes in inflammatory breast cancer treated with antiangiogenic therapy.

Region-of-interest selection is important for quantification of cancer response to therapy.

\section{ACKNOWLEDGMENT}

The Brix and heuristic computer models were provided as programs written in the interactive display language courtesy of Jianhua Yao, PhD.

\section{REFERENCES}

1. Padhani AR. Dynamic contrast-enhanced MRI in clinical oncology: current status and future directions. J Magn Reson Imaging 2002;16:407-422.

2. Miller JC, Pien HH, Sahani D, Sorensen AG, Thrall JH. Imaging angiogenesis: applications and potential for drug development. J Natl Cancer Inst 2005;97:172- 187.

3. Knopp MV, Giesel FL, Marcos H, von Tengg-Kobligk H, Choyke P. Dynamic contrast-enhanced magnetic resonance imaging in oncology. Top Magn Reson Imaging 2001;12: 301-308.

4. Ferrara N, Davis-Smyth T. The biology of vascular endothelial growth factor. Endocr Rev 1997;18:4-25.

5. Borgstrom P, Gold DP, Hillan KJ, Ferrara N. Importance of VEGF for breast cancer angiogenesis in vivo: implications from intravital microscopy of combination treatments with an anti-VEGF neutralizing monoclonal antibody and doxorubicin. Anticancer Res 1999; 19:4203-4214.

6. Taylor JS, Tofts PS, Port R, et al. MR imaging of tumor microcirculation: promise for the new millennium. J Magn Reson Imaging 1999;10:903-907. 
7. Knopp MV, Weiss E, Sinn HP, et al. Pathophysiologic basis of contrast enhancement in breast tumors. J Magn Reson Imaging 1999; 10:260-266.

8. Brix G, Kiessling F, Lucht R, et al. Microcirculation and microvasculature in breast tumors: pharmacokinetic analysis of dynamic MR image series. Magn Reson Med 2004;52:420-429.

9. Kety SS. The theory and applications of the exchange of inert gas at the lungs and tissues. Pharmacol Rev 1951;3:1-41.

10. Brasch R, Pham C, Shames D, et al. Assessing tumor angiogenesis using macromolecular MR imaging contrast media. J Magn Reson Imaging 1997;7:68-74.

11. Gossmann A, Helbich TH, Mesiano S, et al. Magnetic resonance imaging in an experimental model of human ovarian cancer demonstrating altered microvascular permeability after inhibition of vascular endothelial growth factor. Am J Obstet Gynecol 2000; 183:956-963.

12. Wedam SB, Low JA, Yang SX, et al. Antiangiogenic and antitumor effects of bevacizumab in inflammatory and locally advanced breast cancer patients. J Clin Oncol 2006;24: 769-777.

13. Green P. Greene FL, ed. AJCC cancer staging handbook. Berlin, Germany: Springer, 2002.

14. Therasse P, Arbuck SG, Eisenhauer EA, et al; for the European Organization for Research and Treatment of Cancer, National Cancer Institute of the United States, and National Cancer Institute of Canada. New guidelines to evaluate the response to treatment in solid tumors. J Natl Cancer Inst 2000;92:205-216.

15. Port RE, Knopp MV, Hoffmann U, Milker-Zabel S, Brix G. Multicompartment analysis of gadolinium chelate kinetics: blood-tissue exchange in mammary tumors as monitored by dynamic MR imaging. J Magn Reson Imaging 1999;10:233-241.

16. Renkin EM. Transport of potassium-42 from blood to tissue in isolated mammalian skeletal muscles. Am J Physiol 1959;197:1205- 1210.

17. Crone $\mathrm{C}$. The permeability of capillaries in various organs as determined by use of the 'indicator diffusion' method. Acta Physiol Scand 1963;58:292-305.

18. Gribbestad IS, Nilsen G, Fjosne HE, Kvinnsland S, Haugen OA, Rinck PA. Comparative signal intensity measurements in dynamic gadolinium-enhanced MR mammography. J Magn Reson Imaging 1994;4: 477-480.

19. Mussurakis S, Gibbs P, Horsman A. Primary breast abnormalities: selective pixel sampling on dynamic gadolinium-enhanced MR images. Radiology 1998;206:465-473.

20. Galbraith SM, Lodge MA, Taylor NJ, et al. Reproducibility of dynamic contrastenhanced MRI in human muscle and tumours: comparison of quantitative and semi-quantitative analysis. NMR Biomed 2002;15:132- 142.

21. Jackson A, Jayson GC, Li KL, et al. Reproducibility of quantitative dynamic contrast-enhanced MRI in newly presenting glioma. Br J Radiol 2003;76:153162.

22. Pickles MD, Lowry M, Manton DJ, Gibbs P, Turnbull LW. Role of dynamic contrast enhanced MRI in monitoring early response of locally advanced breast cancer to neoadjuvant chemotherapy. Breast Cancer Res Treat 2005;91:1-10.

23. Liney GP, Gibbs P, Hayes C, Leach MO, Turnbull LW. Dynamic contrastenhanced MRI in the differentiation of breast tumors: user-defined versus semiautomated region-of-interest analysis. J Magn Reson Imaging 1999;10:945-949. 

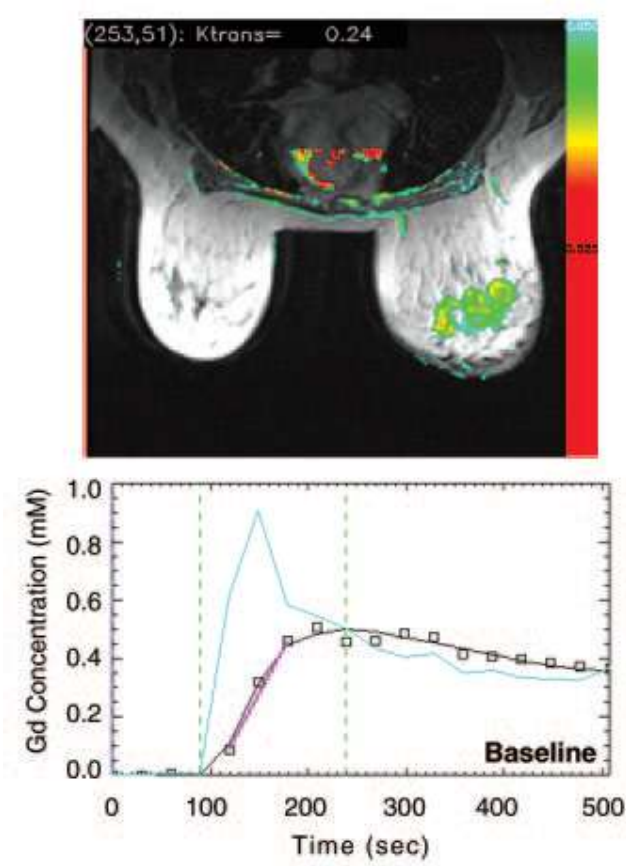

$\begin{array}{ll}\text { ROI } & \text { Current time } \\ \text { Arrival (LMB) /Peak (RMB) } & \text { Alln }\end{array}$
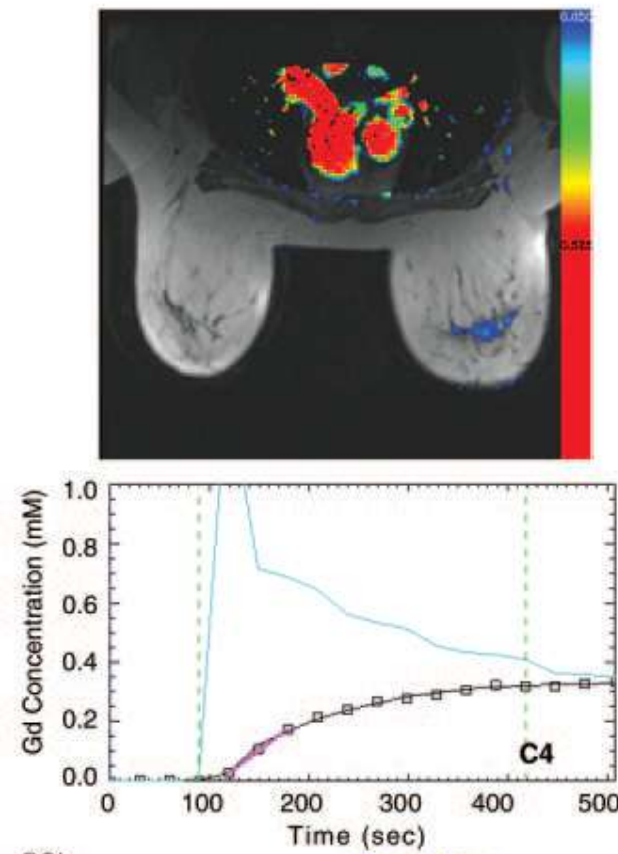

ROI

Arrival (LMB) /Peak (RMB)
Current time
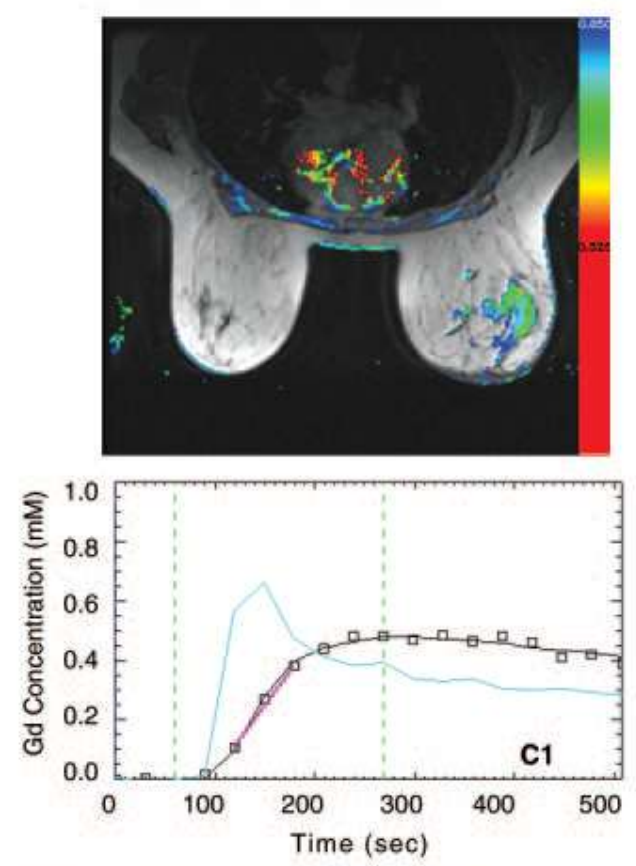

$\begin{array}{ll}\text { ROI } & \text { Current time } \\ \text { Arrival (LMB) /Peak (RMB) } & \text { Alfn }\end{array}$
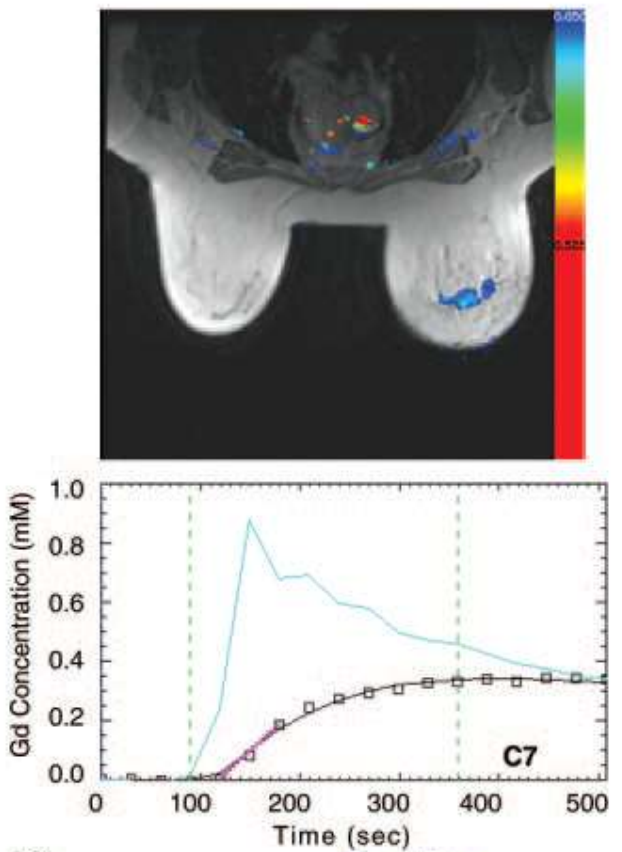

$\mathrm{ROI}$

Arrival (LMB) /Peak (AMB)

Current time

Figure 1. Change in serial transverse GKM $\mathrm{K}^{\text {trans }}$ parametric maps (calculated from transverse T1-weighted spoiled gradient-echo sequence [8/4.2, $25^{\circ}$ flip angle, 4-5-mm section thickness]) (images at top) and gadolinium (Gd) concentration-time curves (graphs at bottom) for one patient from baseline to cycle 7 (C7). Tumor enhancement in the involved breast can be seen in color: Red and green indicate high enhancement, and blue indicates low enhancement. Gadolinium concentration-time curves show the rate of gadolinium-based contrast material perfusion throughout the tumor. Blue line represents arterial input function (AIfn). $\square=$ ROI data, $\mathrm{C} 1=$ cycle $1, \mathrm{C} 4=$ cycle 4 , $\mathrm{LMB}=$ left mouse button, $\mathrm{RMB}=$ right mouse button. 


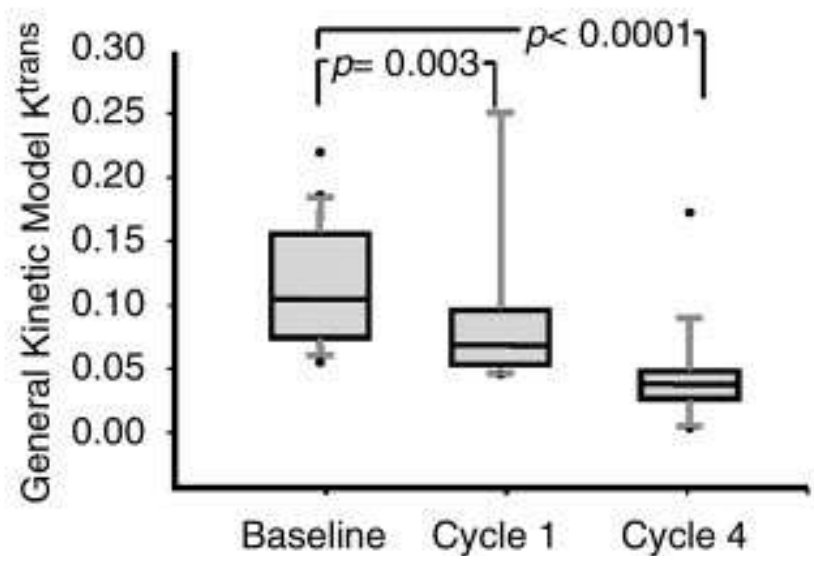

Figure 2. Graph illustrates absolute decreases in $K^{\text {trans }}$ from baseline to cycles 1 and 4 . Two-sided $P$ values were calculated with the Wilcoxon signed rank test $(P=.003$ for difference in $K^{\text {trans }}$ between cycle 1 and baseline, $P<.001$ for difference between cycle 4 and baseline). The horizontal line inside each box is the median quartile, the horizontal line below the box is the lower quartile, and the line above the box is the upper quartile. The vertical lines connect the quartiles.

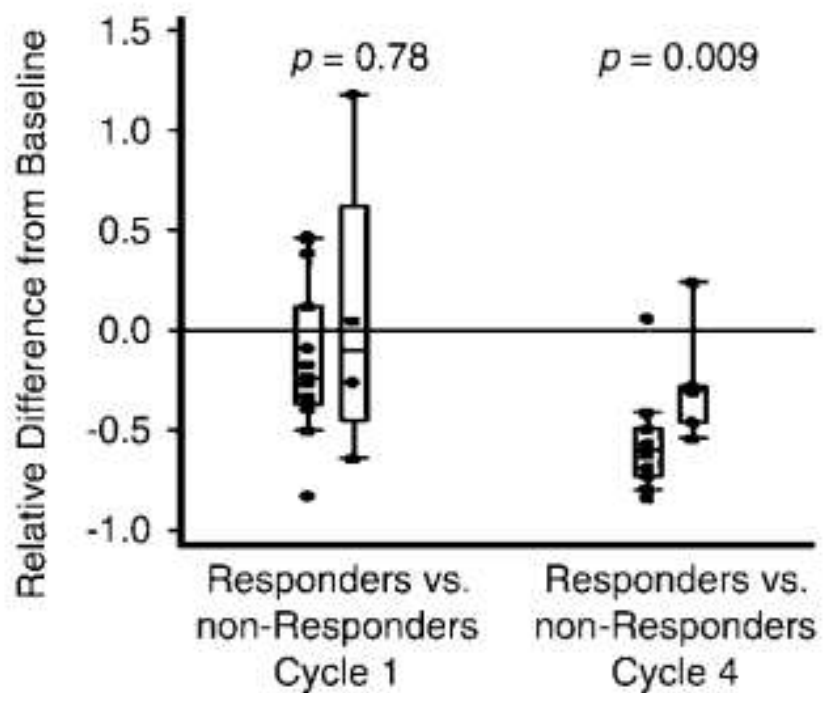

Figure 3. Graph illustrates differences in relative percentage change in slope wash-in from baseline between responders (patients with partial response to therapy) and nonresponders (patients with stable or progressive disease after therapy) (outliers not shown). Response was defined according to the RECIST guidelines. Two-sided $P$ values were calculated by using Wilcoxon rank sum tests. The horizontal line inside each box is the median quartile, the horizontal line below the box is the lower quartile, and the line above the box is the upper quartile. The vertical lines connect the quartiles. 


\begin{tabular}{|c|c|c|c|c|c|c|}
\hline \multirow{2}{*}{ Parameter } & \multicolumn{2}{|c|}{ Baseline to Cycle 1} & \multicolumn{2}{|c|}{ Baseline to Cycle 4} & \multicolumn{2}{|c|}{ Baseline to Cycle 7} \\
\hline & $\begin{array}{c}\text { Median Percentage } \\
\text { Change* }^{*}\end{array}$ & $P$ Value $^{\dagger}$ & $\begin{array}{c}\text { Median Percentage } \\
\text { Change* }\end{array}$ & $P$ Value $^{\dagger}$ & $\begin{array}{c}\text { Median Percentage } \\
\text { Change* }\end{array}$ & $\boldsymbol{P}$ Value $^{\dagger}$ \\
\hline Brix amplitude ${ }^{*}$ & $-14(-63$ to 78$)$ & .35 & $-39(-100$ to 65$)$ & $<.001$ & $-39(-100$ to 43$)$ & .003 \\
\hline Brix $K_{\mathrm{ep}}$ & $-12(-65$ to 96$)$ & .15 & $-54(-100$ to 9$)$ & $<.001$ & $-60(-100$ to 11$)$ & $<.001$ \\
\hline GKM $K^{\text {trans }}$ & $-34(-72$ to 889$)$ & .003 & $-58(-95$ to 6$)$ & $<.001$ & -76 (-98 to 2$)$ & $<.001$ \\
\hline $\mathrm{GKM} K_{\mathrm{ep}}$ & $-15(-54$ to 17$)$ & $<.001$ & $-49(-85$ to 57$)$ & .002 & $-59(-99$ to -9$)$ & $<.001$ \\
\hline Slope wash-in & $-24(-83$ to 118$)$ & .35 & $-55(-84$ to 24$)$ & $<.001$ & $-67(-93$ to 16$)$ & $<.001$ \\
\hline Slope washout & $-64(-1147$ to 2133$)$ & .11 & $26(-1700$ to 1511$)$ & .89 & $-103(-3935$ to 271$)$ & .28 \\
\hline IAUGC $90^{\S}$ & $-27(-86$ to 33$)$ & .02 & $-60(-100$ to 0$)$ & $<.001$ & $-75(-100$ to -25$)$ & $<.001$ \\
\hline IAUGC $180^{\S}$ & $-23(-72$ to 38$)$ & .009 & $-53(-93$ to 0$)$ & $<.001$ & $-62(-95$ to -14$)$ & $<.001$ \\
\hline \multicolumn{7}{|c|}{$\begin{array}{l}\text { *Values are percentage relative changes in the given parameter between the two time points indicated. Numbers in } \\
\text { parentheses are ranges. } \\
\dagger \text { Two-sided } P \text { values calculated with the Wilcoxon signed rank test. } \\
\text { Brix model amplitude of enhancement. } \\
\S \text { IAUGC at } 90 \text { or } 180 \text { seconds after contrast material injection. }\end{array}$} \\
\hline
\end{tabular}

\begin{tabular}{|c|c|c|c|c|}
\hline \multicolumn{5}{|c|}{$\begin{array}{l}\text { Table 2. Relative Changes in Eight Parameters from Cycle } 1 \text { to Cycle } 4 \text { and from } \\
\text { Cycle } 4 \text { to Cycle } 7\end{array}$} \\
\hline \multirow[b]{2}{*}{ Parameter } & \multicolumn{2}{|c|}{ Cycle 1 to Cycle 4} & \multicolumn{2}{|c|}{ Cycle 4 to Cycle 7} \\
\hline & $\begin{array}{l}\text { Median Percentage } \\
\text { Change* }\end{array}$ & $P$ Value $\dagger$ & $\begin{array}{c}\text { Median } \\
\text { Percentage } \\
\text { Change }^{*}\end{array}$ & $P$ Value $\uparrow$ \\
\hline Brix amplitude & $-33(-100$ to 65$)$ & .003 & $2(-100$ to 120$)$ & .46 \\
\hline Brix $K_{\text {ep }}$ & $-45(-100$ to 78$)$ & .002 & $-13(-100$ to 203$)$ & .86 \\
\hline GKM $K^{\text {trans }}$ & $-58(-97$ to 80$)$ & .01 & $-12(-85$ to 240$)$ & .76 \\
\hline GKM $K_{\text {ep }}$ & $-47(-84$ to 134$)$ & .05 & $-14(-96$ to 296$)$ & .89 \\
\hline Slope wash-in & $-47(-81$ to 245$)$ & .02 & $-10(-83$ to 180$)$ & .39 \\
\hline Slope washout & $-28(-1017$ to 2806$)$ & .96 & $-38(-519$ to 228$)$ & .36 \\
\hline IAUGC $90^{\S}$ & $-50(-100$ to 200$)$ & .12 & $-35(-100$ to 50$)$ & .13 \\
\hline IAUGC $180^{\S}$ & $-44(-94$ to 71$)$ & .007 & $0(-67$ to 50$)$ & .52 \\
\hline \multicolumn{5}{|c|}{$\begin{array}{l}\text { * Values are percentage relative changes between the two time points indicated. Numbers in } \\
\text { parentheses are ranges. } \\
\dagger \text { Two-sided } P \text { values calculated with the Wilcoxon signed rank test. } \\
\text { Brix model amplitude of enhancement. } \\
\S \text { IAUGC at } 90 \text { or } 180 \text { seconds after contrast material injection. }\end{array}$} \\
\hline
\end{tabular}


Table 3. Relative Changes in Parameters for Responders and Nonresponders

\begin{tabular}{|c|c|c|c|c|c|c|}
\hline \multirow[b]{2}{*}{$\begin{array}{l}\text { Parameter and } \\
\text { Patient Group* }\end{array}$} & \multicolumn{3}{|c|}{ Baseline to Cycle 1} & \multicolumn{3}{|c|}{ Baseline to Cycle 4} \\
\hline & $\begin{array}{c}\text { No. of } \\
\text { Patients }\end{array}$ & $\begin{array}{c}\text { Median } \\
\text { Percentage } \\
\text { Change }^{\dagger} \\
\end{array}$ & $\begin{array}{c}P \\
\text { Value }^{*}\end{array}$ & $\begin{array}{c}\text { No. of } \\
\text { Patients }\end{array}$ & \begin{tabular}{|c|} 
Median \\
Percentage \\
Change $^{\dagger}$ \\
\end{tabular} & $\begin{array}{c}P \\
\text { Value }^{\ddagger}\end{array}$ \\
\hline Brix amplitude ${ }^{\S}$ & & & .57 & & & .01 \\
\hline Partial responders & 13 & $-12(-28$ to 47$)$ & & 13 & $-53(-100$ to -12$)$ & \\
\hline Nonresponders & 5 & $-26(-63$ to 78$)$ & & 6 & $-22(-39$ to 65$)$ & \\
\hline Brix $K_{\mathrm{ep}}$ & & & .96 & & & .34 \\
\hline Partial responders & 12 & $-19(-65$ to 96$)$ & & 12 & $-66(-100$ to 9$)$ & \\
\hline Nonresponders & 5 & $-10(-44$ to -4$)$ & & 6 & $-40(-84$ to -24$)$ & \\
\hline GKM $K^{\text {trans }}$ & & & .77 & & & .05 \\
\hline Partial responders & 13 & $-32(-72$ to 889$)$ & & 13 & $-76(-95$ to 6$)$ & \\
\hline Nonresponders & 5 & $-36(-56$ to -9$)$ & & 6 & $-39(-57$ to -6$)$ & \\
\hline GKM $K_{\mathrm{ep}}$ & & & .34 & & & .09 \\
\hline Partial responders & 13 & $-14(-33$ to 17$)$ & & 13 & $-61(-85$ to 57$)$ & \\
\hline Nonresponders & 5 & $-16(-54$ to -2$)$ & & 6 & $-24(-59$ to 50$)$ & \\
\hline Slope wash-in & & & .78 & & & .009 \\
\hline Partial responders & 13 & $-24(-83$ to 47$)$ & & 13 & $-60(-84$ to 6$)$ & \\
\hline Nonresponders & 4 & $-11(-64$ to 118$)$ & & 6 & $-31(-55$ to 24$)$ & \\
\hline Slope washout & & & .02 & & & .06 \\
\hline Partial responders & 13 & $-104(-1147$ to 535$)$ & & 13 & $-181(-1700$ to 400$)$ & \\
\hline Nonresponders & 4 & $65(-25$ to 2133$)$ & & 6 & $68(26$ to 1511$)$ & \\
\hline IAUGC 90" & & & .04 & & & .24 \\
\hline Partial responders & 13 & $-14(-67$ to 33$)$ & & 13 & $-67(-100$ to 0$)$ & \\
\hline Nonresponders & 5 & $-60(-86$ to 0$)$ & & 6 & $-54(-71$ to -25$)$ & \\
\hline IAUGC $180^{\#}$ & & & .08 & & & .04 \\
\hline Partial responders & 13 & $-18(-35$ to 38$)$ & & 13 & $-61(-93$ to 0$)$ & \\
\hline Nonresponders & 5 & $-42(-72$ to 21$)$ & & 6 & $-40(-53$ to -12$)$ & \\
\hline \multicolumn{7}{|c|}{$\begin{array}{l}\text { * Nonresponders are patients with stable or progressive disease after therapy. } \\
\dagger \text { Values are percentage relative changes between the two time points indicated. Numbers in } \\
\text { parentheses are ranges. } \\
\$ \text { Two-sided P values calculated with the Wilcoxon signed rank test. } \\
\S \text { Brix model amplitude of enhancement. } \\
\text { \# IAUGC at } 90 \text { or } 180 \text { seconds after contrast material injection. }\end{array}$} \\
\hline
\end{tabular}

